\title{
PHYSICAL PROPERTIES OF AIGaAs EPILAYERS SUBJECTED TO HIGH PRESSURE - HIGH TEMPERATURE TREATMENT
}

\author{
W. Szuszkiewicz ${ }^{a}$, W. GęBICKI ${ }^{b}$, J. BĄK-Misiuk ${ }^{a}$, J. Domagala $^{a}$, \\ M. LESZCZYŃSKI ${ }^{c}$ AND J. HÄRTWIG ${ }^{d}$ \\ ${ }^{a}$ Institute of Physics, Polish Academy of Sciences \\ Al. Lotników 32/46, 02-668 Warsaw, Poland \\ ${ }^{b}$ Warsaw Technical University, Koszykowa 75, 00-662 Warsaw, Poland \\ ${ }^{c}$ High Pressure Research Center, Polish Academy of Sciences, Warsaw, Poland \\ ${ }^{d}$ European Synchrotron Radiation Facility, BP220, 38043 Grenoble Cedex, France
}

\begin{abstract}
AlGaAs layers grown by molecular beam epitaxy on GaAs substrates were investigated before and after high hydrostatic pressure $(1.2 \mathrm{GPa})$ at high temperature $(770 \mathrm{~K})$ treatment. In order to study the influence of high pressure - high temperature treatment on the physical properties of the AlGaAs layers, X-ray, electron transport and Raman scattering measurements were performed at room temperature. The observed changes in the lattice parameter, Raman spectra and free-carrier concentration were related to the strain relaxation and explained by the creation of misfit dislocations and other extended defects which were visible on the synchrotron X-ray topographs after high pressure - high temperature treatment.
\end{abstract}

PACS numbers: 81.40.-z, 73.30.+y, 73.60.Br

\section{Introduction}

It is established that $\mathrm{AlGaAs}$ layers change their unit cell volume after high pressure - high temperature treatment (HP - HT) [1]. The observed changes of the lattice,parameter are related to the creation of defects, which can be detected by X-ray synchrotron topographs. This effect is due to enhanced strain at precipitate/matrix boundary caused by high pressure.

The question arises if HP - HT treatment has a noticeable influence on the basic physical properties of the material under consideration. The comparison of selected optical and transport properties of the same layers before and after HP - HT treatment were carried out. It is well known that the phonon frequency of a given semiconductor depends on the lattice parameter, so it was a natural choice to investigate the lattice excitations of the system. There exist two kinds of the optical measurements which could be applied for such investigations: infrared reflectivity measurements in the restrahlen spectral range and the Raman 
scattering measurements. The second method should be much better in our case, because both optical modes (TO and LO) may be directly seen in the Raman scattering spectra. Moreover, in the presence of a high concentration of free carriers in the investigated samples, the reflectivity spectra of phonon-related structures are modificated by plasma effects and it could be difficult to interpret them qualitatively. Taking into account the above-mentioned arguments, the lattice dynamics was investigated by the Raman scattering.

AlGaAs mixed crystals exhibit the two-mode behaviour, i.e., in the optical phonon excitations spectra, two separate branches (resulting from GaAs-like and AlAs-like optical modes) are observed. The LO and TO phonon frequency dependence on the mixed crystal composition is relatively well known. It was measured in the past by both infrared reflection and Raman scattering measurements. There exists a slight scattering of the experimental values of the optical phonon frequency for a given mixed crystal composition (the accuracy of the room temperature measurements is not so high as of those performed at low temperatures). Nevertheless, on the basis of the optical modes frequency measurements the mixed crystal composition can be determined with an accuracy of a few percent. One can find a short review concerning this problem, e.g., in Ref. [3].

\section{Experimental}

$3 \mu \mathrm{m}$ thick $\mathrm{Al}_{0.27} \mathrm{Ga}_{0.73} \mathrm{As}$ layers grown by molecular beam epitaxy (MBE) at temperature of about $1000 \mathrm{~K}$ on the LEC-grown (liquid encapsulated Czochralski) GaAs substrates were subjected to HP (1.2 GPa) at IIT (770 K) for 1 hour. The HP - HT annealings were carried out in a pressure furnace lined by $\mathrm{Al}_{2} \mathrm{O}_{3}$ and filled with purified argon gas. The pressure was maintained constant during the annealing time within $2 \%$ and the temperature within $\pm 2^{\circ} \mathrm{C}$. The error of absolute pressure value was about $20 \mathrm{MPa}$. Samples were cooled after the treatment (the cooling rate was about $2 \mathrm{Ks}^{-1}$ ) and HP was releasing to ambient.

An influence of HP - HT treatment on the properties of the AlGaAs/GaAs system was studied [1] by lattice parameter measurements using the high resolution diffractometer and the Bond method as well as by X-ray synchrotron topography.

We performed the Raman scattering measurements for the AlGaAs epilayers in order to compare the results obtained for as-grown samples and the sample subjected to the HP - HT treatment. Raman scattering measurements were done at room temperature in a quasi backscattering geometry. Spectra were recorded using the DILOR XY Raman spectrometer with CCD detecting system. An $\mathrm{Ar}^{+}$ laser line (with the wavelength equal to $514.5 \mathrm{~nm}$ ) was used for the excitation.

The free-carrier concentration and Hall mobility measurements were performed at room temperature by the van der Pauw method [2]. Pure indium was applied in order to make the electrical contacts for AlGaAs epilayers.

\section{Results and discussion}

No change of the lattice parameter value for GaAs substrate due to the HP - HT treatment was found by the Bond measurements so probably the possible process of $\mathrm{Al}$ diffusion from AlGaAs epilayer to GaAs substrate could be neglected. The lattice parameters parallel to the interface, $a_{\|}$, for all investigated layers were 
found to be equal to the lattice parameters of GaAs substrate which means that the layers are strained. On the contrary the lattice parameter perpendicular to the interface, $a_{\perp}$, was higher for the sample after the HP - HT treatment than that for as-grown sample. This difference was equal to $\Delta a_{\perp}=5 \times 10^{-4}$ [1]. The changes of lattice parameters of the AlGaAs layers after the HP - HT treatment were related to the strain relaxation and explained by the creation of misfit dislocations and other extended defects visible on the topographs. As it is seen on the transmission topographs HP - IIT processed sample contains a high concentration of defects, probably inclusions or precipitates. Moreover, the misfit dislocation lines (parallel to the [011] direction) were observed for this sample (topographs of as-grown sample show no misfit dislocation but only a cellular structure of dislocations, that is typical for LEC-grown GaAs substrates). The comparison of topographs taken for as-grown and IIP - HT treated samples are shown in Fig. 1.
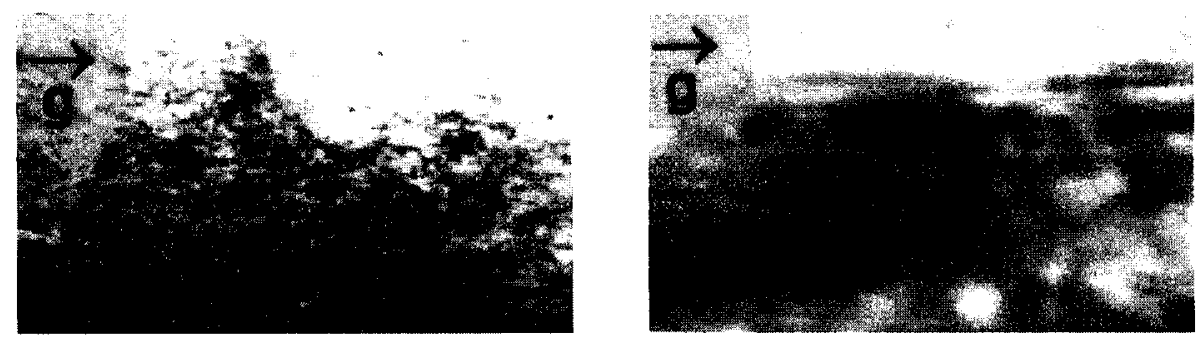

Fig. 1. Transmission topographs of as-grown (a) and HP - HT treated (b) samples taken for diffraction vector $g=[022]$ and X-ray wavelength $\lambda=0.39 \AA$. Scale and $g$ are marked in the figure. Magnification $120 \times$.

Lattice dynamical behaviour of the material reflects the changes of lattice parameters and disorder of the crystal structure. Raman spectra presented in Fig. 2 show some differences in the phonon band intensity between the as-grown samples and those obtained after the IIP - IIT treatment. We observed that after the treatment there is a considerable increase in intensity of disorder activated longitudinal acoustic (DALA) phonon from the border of the Brillouin zone. The intensity of DALA phonon is a good measure of the lack of translational symmetry. At the same time we detected an increase in the LO phonon intensity for the sample after the HP - HT treatment. We attribute this effect to the relaxation of the Raman selection rules due to a short-range lattice disorder. These results are in a good agreement with the X-ray results discussed previously [1]. A shift of the optical phonon energy is observed. This effect may be easily explained as a consequence of the change of the lattice parameters. The shift of the optical phonon frequency is quite well defined for GaAs-like phonons (about $3 \mathrm{~cm}^{-1}$ ). For AlAs-like phonon this shift is estimated to be as high as $c a .5 \mathrm{~cm}^{-1}$.

The results of measurements of the free-carrier concentration and Hall mobility obtained for the examined samples, due to their small size, possess rather large experimental errors. Nevertheless, it was determined that all investigated 


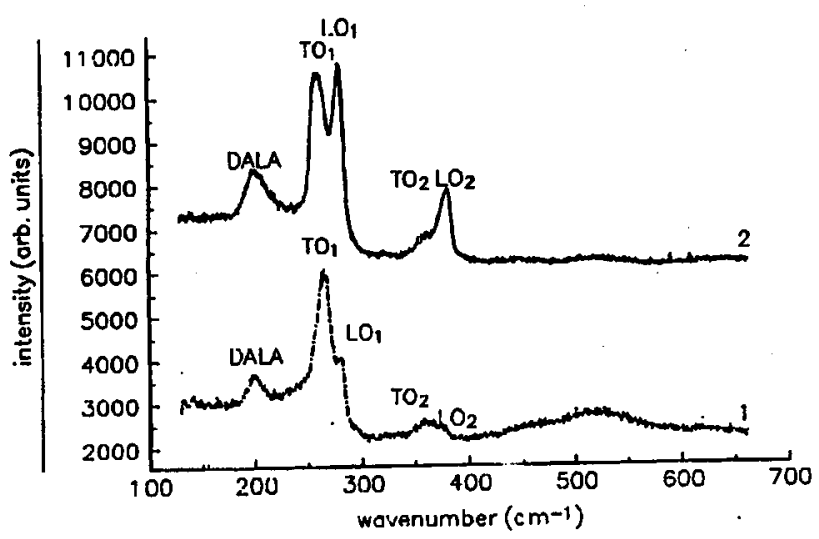

Fig. 2. Raman spectra of $\mathrm{Al}_{0.27} \mathrm{Ga}_{0.73} \mathrm{As} / \mathrm{GaAs}$ samples: (1) as-grown, (2) after HP - HT treatment.

layers are of $n$-type. The initial electron concentration was of the order of about $(3 \pm 1) \times 10^{17} \mathrm{~cm}^{-3}$. After the HP - HT treatment the free-electron concentration decreases by about twice. Moreover, a significant decrease in the electron mobility was observed in this case (to $1 / 20$-th of the initial value equal to about $500 \mathrm{~cm}^{2} /(\mathrm{V} \mathrm{s})$ ). In our opinion, both effects (decrease in both the electron concentration and electron mobility) result from the creation of a large number of point and extended defects. As it was mentioned above a large number of misfit dislocations and the other extended defects have been observed by the X-ray topographs of the HP - HT treated samples. These charged defects can act as the electron traps and due to this the free-carrier concentration decreases. Moreover, a part of these defects could be multicharged Coulomb centers, which can act as very effective electron scattering centers. Such a hypothesis is able to explain a dramatic decrease in the measured electron mobility.

\section{Conclusions}

In conclusion, we have reported that the microstructural change of the layers due to the HP - HT treatment strongly influences the optical and transport properties. The following effects produced by the HP - HT treatment of AlGaAs are observed:

(1) Change of the lattice parameters and of the concentrations of misfit dislocations and other extended defects;

(2) Strong change of free carrier mobility and less pronounced decrease in free-carrier concentration;

(3) Considerable effects visible in Raman scattering experiment, i.e., strong increase in the LO phonon intensity in the HP - HT treated sample and of the disorder-activated LA phonon mode. A small shift of the phonon energies due to the lattice strain relaxation is detected.

We conclude that X-ray diffraction, transport measurements and Raman scattering are complementary tools for characterization of the HP - HT treatment influence on the physical properties of AlGaAs. 


\section{Acknowledgments}

We would like to express our gratitude to Dr J. Marczewski and Dr J. Kaniewski for performing the electron transport measurements.

This work was supported in part by the Committee for Scientific Research (Poland) grant No. 101/P3/93/05 (2 P302 083 05). A part of activities was made within the Polish-French Scientific and Technological Cooperation Joint, project No. 6197. The Philips MRD diffractometer was founded by the Foundation for Polish Science (program SEZAM 94).

\section{References}

[1] J. Bąk-Misiuk, J. Domagała, J. Trela, M. Leszczyński, A. Misiuk, J. Härtwig, E. Prieur, Acta Phys. Pol. A 89, 405 (1996).

[2] L.J. van der Pauw, Philips Res. Repts. 13, 1 (1958).

[3] Properties of Aluminium Gallium Arsenide, Ed. S. Adachi, INSPEC, Institution of Electrical Engineers, London 1993. 